Supporting information for:

\title{
Highly Stereoselective Aldol Reactions of Titanium Enolates from Lactate-Derived Chiral Ketones
}

Joan G. Solsona, Pedro Romea,* Fèlix Urpí,* Jaume Vilarrasa

\section{Characterisation data for compounds 3,5 , and 6.}

1. (2S,4S,5R)-5-Hydroxy-2-(p-methoxybenzyloxy)-4,6-dimethyl-3-heptanone (3a).

Colourless oil. $\boldsymbol{R}_{f}\left(\right.$ hexanes/EtOAc 85:15) $=0.2$. HPLC $($ hexanes $/ i-\mathrm{PrOH} 99: 1) \boldsymbol{t}_{\mathbf{R}}=13.3 \mathrm{~min}$. $[\boldsymbol{\alpha}]_{\mathrm{D}}=$ -18.5 ( $c=1.78, \mathrm{CHCl}_{3}$ ). IR (film): v 3519 (br), 2961, 2939, 1710, 1515, 1250, $1111 \mathrm{~cm}^{-1} .{ }^{1} \mathbf{H} \mathbf{~ N M R}$

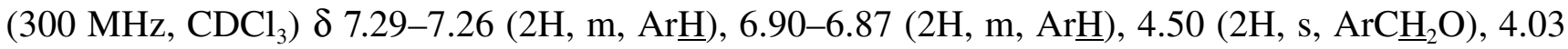
$(1 \mathrm{H}, \mathrm{q}, J=6.7, \mathrm{C} \underline{\mathrm{HOPMB}}), 3.81\left(3 \mathrm{H}, \mathrm{s}, \mathrm{OC}_{3}\right), 3.45(1 \mathrm{H}, \mathrm{dd}, J=8.2, J=2.9, \mathrm{C} \underline{\mathrm{HOH}}), 3.22(1 \mathrm{H}, \mathrm{qd}$, $\left.J=7.0, J=2.9, \mathrm{COCHCH}_{3}\right), 1.72-1.60\left(1 \mathrm{H}, \mathrm{m}, \mathrm{CH}_{3} \mathrm{CHCH}_{3}\right), 1.35\left(3 \mathrm{H}, \mathrm{d}, J=6.7, \mathrm{C}_{3} \mathrm{CHOPMB}\right)$, $1.07\left(3 \mathrm{H}, \mathrm{d}, J=7.0, \mathrm{COCHC}_{3}\right), 1.00\left(3 \mathrm{H}, \mathrm{d}, J=6.6, \mathrm{C}_{3} \mathrm{CHCH}_{3}\right), 0.85(3 \mathrm{H}, \mathrm{d}, J=6.9$, $\left.\mathrm{CH}_{3} \mathrm{CHCH}_{3}\right) .{ }^{13} \mathrm{C}$ NMR $\left(75.4 \mathrm{MHz}, \mathrm{CDCl}_{3}\right) \delta 217.0,159.4,129.5,113.9,79.0,76.4,71.4,55.3,42.6$, 30.7, 19.1, 19.0, 16.7, 9.3. HRMS (+FAB): $m / z$ calcd. for $\mathrm{C}_{17} \mathrm{H}_{27} \mathrm{O}_{4}[\mathrm{M}+\mathrm{H}]^{+}:$295.1909. Found: 295.1915.

2. (2S,4S,5R)-5-Hydroxy-2-( $p$-methoxybenzyloxy)-4,7-dimethyl-3-octanone (3b).

Colourless oil. $\boldsymbol{R}_{\boldsymbol{f}}$ (hexanes/EtOAc 80:20) $=0.2$. HPLC (hexanes $/ i$-PrOH 99:1) $\boldsymbol{t}_{\mathbf{R}}=15.5 \mathrm{~min} .[\boldsymbol{\alpha}]_{\mathbf{D}}=$ -3.7 (c = 1.39, $\mathrm{CHCl}_{3}$ ). IR (film): v 3504 (br), 2956, 2871, 1717, 1515, 1250, $1036 \mathrm{~cm}^{-1} .{ }^{1} \mathbf{H} \mathbf{~ N M R}$ $\left(300 \mathrm{MHz}, \mathrm{CDCl}_{3}\right) \delta$ 7.29-7.26 (2H, m, Ar$\left.\underline{\mathrm{H}}\right), 6.90-6.87(2 \mathrm{H}, \mathrm{m}, \mathrm{Ar} \underline{\mathrm{H}}), 4.50\left(2 \mathrm{H}, \mathrm{s}, \mathrm{ArC}_{2} \mathrm{O}\right), 4.03$ $(1 \mathrm{H}, \mathrm{q}, J=6.7, \mathrm{C} \underline{\mathrm{HOPMB}}), 3.93-3.91(1 \mathrm{H}, \mathrm{m}, \mathrm{C} \underline{\mathrm{HOH}}), 3.81\left(3 \mathrm{H}, \mathrm{s}, \mathrm{OC}_{3}\right), 3.01(1 \mathrm{H}, \mathrm{qd}, J=7.0, J$ = 3.0, $\left.\mathrm{COC}^{\mathrm{HCH}}{ }_{3}\right), 1.82-1.68\left(1 \mathrm{H}, \mathrm{m}, \mathrm{CH}_{3} \underline{\mathrm{HCH}}_{3}\right), 1.48-1.39\left(1 \mathrm{H}, \mathrm{m}, \mathrm{CH}(\mathrm{OH}) \mathrm{C}_{\mathrm{x}} \mathrm{H}_{\mathrm{y}}\right), 1.35(3 \mathrm{H}, \mathrm{d}$, $\left.J=6.7, \mathrm{C}_{3} \mathrm{CHOPMB}\right), 1.14-1.05\left(1 \mathrm{H}, \mathrm{m}, \mathrm{CH}(\mathrm{OH}) \mathrm{CH}_{\mathrm{x}} \underline{\mathrm{H}}_{\mathrm{y}}\right), 1.08\left(3 \mathrm{H}, \mathrm{d}, J=7.0, \mathrm{COCHC} \underline{H}_{3}\right), 0.91$ $\left(3 \mathrm{H}, \mathrm{d}, J=6.7, \mathrm{C}_{3} \mathrm{CHCH}_{3}\right), 0.90\left(3 \mathrm{H}, \mathrm{d}, J=6.7, \mathrm{CH}_{3} \mathrm{CHC}_{3}\right) .{ }^{13} \mathbf{C} \mathbf{N M R}\left(75.4 \mathrm{MHz}, \mathrm{CDCl}_{3}\right) \delta$ $216.7,159.5,129.5,113.9,79.1,71.4,69.3,55.3,45.6,43.2,24.6,23.4,21.9,16.5$, 9.9. HRMS (+FAB): $m / z$ calcd. for $\mathrm{C}_{18} \mathrm{H}_{29} \mathrm{O}_{4}[\mathrm{M}+\mathrm{H}]^{+}:$309.2066. Found: 309.2079. 


\section{3. (2S,4S,5R)-5-Hydroxy-2-(p-methoxybenzyloxy)-4-methyl-3-heptanone (3c).}

Colourless oil. $\boldsymbol{R}_{f}\left(\right.$ hexanes/EtOAc 80:20) $=0.2$. HPLC $($ hexanes $/ i-\mathrm{PrOH} 99: 1) \boldsymbol{t}_{\mathbf{R}}=18.5 \mathrm{~min} .[\boldsymbol{\alpha}]_{\mathbf{D}}=$ -13.8 ( $c=1.11, \mathrm{CHCl}_{3}$ ). IR (film): v 3494 (br), 2966, 2937, 1711, 1515, 1250, $1036 \mathrm{~cm}^{-1} .{ }^{1} \mathbf{H}$ NMR $\left(300 \mathrm{MHz}, \mathrm{CDCl}_{3}\right) \delta$ 7.30-7.20 (2H, m, Ar$\left.\underline{\mathrm{H}}\right), 6.95-6.85$ (2H, m, Ar$\left.\underline{\mathrm{H}}\right), 4.50\left(2 \mathrm{H}, \mathrm{s} . \mathrm{ArC}_{2} \mathrm{O}\right), 4.03$ $(1 \mathrm{H}, \mathrm{q}, J=6.8, \mathrm{CHOPMB}), 3.81\left(3 \mathrm{H}, \mathrm{s}, \mathrm{OC}_{3}\right), 3.81-3.70(1 \mathrm{H}, \mathrm{m}, \mathrm{CHOH}), 3.07(1 \mathrm{H}, \mathrm{qd}, J=7.1, J=$ 3.0, $\left.\mathrm{COC}^{\mathrm{HCH}}{ }_{3}\right), 1.60-1.30\left(2 \mathrm{H}, \mathrm{m}, \underline{\mathrm{C}}_{2} \mathrm{CH}_{3}\right), 1.35$ (3H, d, $\left.J=6.8, \mathrm{C}_{3} \mathrm{CHOPMB}\right), 1.08$ (3H, d, $J=$ 7.1, $\left.\mathrm{COCHCH}_{3}\right), 0.94\left(3 \mathrm{H}, \mathrm{t}, J=7.4, \mathrm{CH}_{2} \mathrm{CH}_{3}\right) .{ }^{13} \mathbf{C ~ N M R}\left(75.4 \mathrm{MHz}, \mathrm{CDCl}_{3}\right) \delta 216.7,159.5,129.5$, $113.9,79.2,72.9,71.5,55.3,44.9,27.0,16.6,10.5,9.9$.

\section{4. (2S,4S,5S)-5-Hydroxy-2-(p-methoxybenzyloxy)-4,6-dimethyl-6-hepten-3-one (3d).}

Colourless oil. $\boldsymbol{R}_{f}$ (hexanes/EtOAc 85:15) $=0.2$. HPLC (hexanes $\left./ i-\mathrm{PrOH} 99: 1\right) \boldsymbol{t}_{\mathbf{R}}=16.1 \mathrm{~min} .[\alpha]_{\mathbf{D}}=$ -11.4 ( $c=2.27, \mathrm{CHCl}_{3}$ ). IR (film): v 3490 (br), 2981, 2939, 1717, 1613, 1515, 1457, 1250, 1111 $\mathrm{cm}^{-1} .{ }^{1} \mathbf{H}$ NMR $\left(400 \mathrm{MHz}, \mathrm{CDCl}_{3}\right) \delta$ 7.28-7.26 (2H, m, ArH $), 6.90-6.88$ (2H, m, Ar$\left.\underline{\mathrm{H}}\right), 5.07$ (1H, br s, $\left.\mathrm{C}\left(\mathrm{CH}_{3}\right)=\mathrm{C}_{\mathrm{x}} \mathrm{H}_{\mathrm{y}}\right), 4.94\left(1 \mathrm{H}\right.$, br s, $\left.\mathrm{C}\left(\mathrm{CH}_{3}\right)=\mathrm{CH}_{\mathrm{x}} \underline{\mathrm{H}}_{\mathrm{y}}\right), 4.50\left(2 \mathrm{H}, \mathrm{s}, \mathrm{ArC}_{2} \mathrm{O}\right), 4.36(1 \mathrm{H}, \mathrm{br} \mathrm{s}, \mathrm{C} \underline{\mathrm{HOH}}), 4.05$ $\left.(1 \mathrm{H}, \mathrm{q}, J=6.8, \mathrm{C} \underline{\mathrm{HOPMB}}), 3.81\left(3 \mathrm{H}, \mathrm{s}, \mathrm{OC}_{3}\right), 3.22\left(1 \mathrm{H}, \mathrm{qd}, J=7.0, J=3.5, \mathrm{COC}^{\mathrm{HCH}}\right)_{3}\right), 1.68$ $\left(3 \mathrm{H}\right.$, br s, $\left.\mathrm{C}\left(\mathrm{C}_{3}\right)=\mathrm{CH}_{2}\right), 1.37\left(3 \mathrm{H}, \mathrm{d}, J=6.8, \mathrm{C}_{3} \mathrm{CHOPMB}\right), 1.03\left(3 \mathrm{H}, \mathrm{d}, J=7.0, \mathrm{COCHC}_{3}\right) .{ }^{13} \mathrm{C}$ NMR (75.4 MHz, $\left.\mathrm{CDCl}_{3}\right) \delta 216.2,159.4,143.7,129.5,129.4,113.9,111.9,79.2,74.0,71.5,55.3$, 43.2, 19.3, 16.7, 9.6. HRMS (+FAB): $m / z$ calcd. for $\mathrm{C}_{17} \mathrm{H}_{25} \mathrm{O}_{4}[\mathrm{M}+\mathrm{H}]^{+}:$293.1753. Found: 293.1737 .

\section{5. (1S,2S,4S)-1-Hydroxy-4-(p-methoxybenzyloxy)-2-methyl-1-phenyl-3-pentanone (3e).}

Colourless oil. $\boldsymbol{R}_{f}$ (hexanes/EtOAc 85:15) $=0.3$. HPLC (hexanes $\left./ i-\operatorname{PrOH} 99: 1\right) \boldsymbol{t}_{\mathbf{R}}=18.1 \mathrm{~min} .[\alpha]_{\mathbf{D}}=$ +1.3 ( $c=1.55, \mathrm{CHCl}_{3}$ ). IR (film): v 3475 (br), 3033, 2937, 2838, 1717, 1515, 1248, $1034 \mathrm{~cm}^{-1} .{ }^{1} \mathbf{H}$ NMR $\left(300 \mathrm{MHz}, \mathrm{CDCl}_{3}\right) \delta$ 7.32-7.25 (5H, m, ArH) $, 7.22-7.19(2 \mathrm{H}, \mathrm{m}, \mathrm{Ar} \underline{\mathrm{H}}), 6.88-6.85$ (2H, m, $\operatorname{Ar} \underline{\mathrm{H}}), 4.97(1 \mathrm{H}, \mathrm{d}, J=4.7, \mathrm{C} \underline{\mathrm{HOH}}), 4.37\left(1 \mathrm{H}, \mathrm{AB}\right.$ system, $\left.J=11.2, \mathrm{ArC}_{\mathrm{H}} \mathrm{H}_{\mathrm{y}} \mathrm{O}\right), 4.24(1 \mathrm{H}, \mathrm{AB}$ system, $\left.J=11.2, \operatorname{ArCH}_{x} \underline{H}_{y} \mathrm{O}\right), 3.86(1 \mathrm{H}, \mathrm{q}, J=6.8, \mathrm{CHOPMB}), 3.79\left(3 \mathrm{H}, \mathrm{s}, \mathrm{OC}_{3}\right), 3.29(1 \mathrm{H}, \mathrm{qd}, J=$ $\left.7.0, J=4.7, \mathrm{COC}_{\mathrm{HCH}}\right)_{3}, 1.24\left(3 \mathrm{H}, \mathrm{d}, J=6.8, \mathrm{C}_{3} \mathrm{CHOPMB}\right), 1.09\left(3 \mathrm{H}, \mathrm{d}, J=7.0, \mathrm{COCHC}_{3}\right) .{ }^{13} \mathrm{C}$ NMR $\left(75.4 \mathrm{MHz}, \mathrm{CDCl}_{3}\right) \delta 215.9,159.4,141.8,129.6,129.4,128.3,127.5,126.3,113.8,79.7,73.8$, 71.4, 55.3, 48.0, 16.4, 11.1. HRMS (+FAB): $m / z$ calcd. for $\mathrm{C}_{20} \mathrm{H}_{25} \mathrm{O}_{4}[\mathrm{M}+\mathrm{H}]^{+}:$329.1753. Found: 329.1763 . 
6. (1S,2S,4S)-1-(p-Chlorophenyl)-1-hydroxy-4-(p-methoxybenzyloxy)-2-methyl-3-pentanone (3f). White solid. $\mathbf{m p}=71-73{ }^{\circ} \mathrm{C} . \boldsymbol{R}_{f}$ (hexanes/EtOAc 85:15) $=0.2$. HPLC (hexanes $\left./ i-\operatorname{PrOH} 99: 1\right) \boldsymbol{t}_{\mathbf{R}}=$ 20.0 min. $[\alpha]_{\mathbf{D}}=-7.3\left(c=1.58, \mathrm{CHCl}_{3}\right)$. IR (film): v 3467 (br), 2981, 2937, 1717, 1613, 1515, 1250,

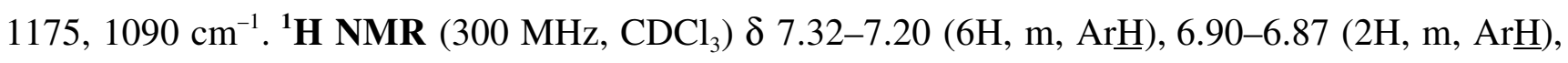
$4.98(1 \mathrm{H}, \mathrm{dd}, J=4.2, J=1.8, \mathrm{C} \underline{\mathrm{HOH}}), 4.43\left(1 \mathrm{H}, \mathrm{AB}\right.$ system, $\left.J=11.3, \mathrm{ArC}_{\mathrm{H}} \mathrm{H}_{\mathrm{y}} \mathrm{O}\right), 4.37(1 \mathrm{H}, \mathrm{AB}$ system, $\left.J=11.3, \mathrm{ArCH}_{\mathrm{x}} \underline{\mathrm{H}}_{\mathrm{y}} \mathrm{O}\right), 3.92(1 \mathrm{H}, \mathrm{q}, J=6.9, \mathrm{C} \underline{\mathrm{HOPMB}}), 3.81\left(3 \mathrm{H}, \mathrm{s}, \mathrm{OC}_{3}\right), 3.27(1 \mathrm{H}, \mathrm{qd}, J=$ $\left.4.2, J=7.0, \mathrm{COC}_{\underline{H C H}}\right), 1.28\left(3 \mathrm{H}, \mathrm{d}, J=6.9, \mathrm{C}_{3} \mathrm{CHOPMB}\right), 1.04\left(3 \mathrm{H}, \mathrm{d}, J=7.0, \mathrm{COCHC}_{3}\right) .{ }^{13} \mathbf{C}$ NMR $\left(75.4 \mathrm{MHz}, \mathrm{CDCl}_{3}\right) \delta 216.1,159.5,140.2,133.2,129.5,128.4,127.6,113.9,79.4,72.9,71.5$, $55.3,47.6,16.3,10.5$.

\section{7. (2S,4S,5R)-2-Benzyloxy-5-hydroxy-4,6-dimethyl-3-heptanone (5a).}

Colourless oil. $\boldsymbol{R}_{f}($ hexanes/EtOAc 85:15) $=0.2$. HPLC (hexanes $/ i-\mathrm{PrOH} 99: 1) \boldsymbol{t}_{\mathbf{R}}=8.7 \mathrm{~min} .[\boldsymbol{\alpha}]_{\mathbf{D}}=$ -16.0 ( $c=1.06, \mathrm{CHCl}_{3}$ ). IR (film): v 3512 (br), 2964, 2875, 1709, 1457, $1117 \mathrm{~cm}^{-1}$. ${ }^{1} \mathbf{H}$ NMR (500 $\left.\mathrm{MHz}, \mathrm{CDCl}_{3}\right) \delta 7.40-7.30(5 \mathrm{H}, \mathrm{m}, \mathrm{Ar} \underline{\mathrm{H}}), 4.57\left(2 \mathrm{H}, \mathrm{s}, \mathrm{PhC}_{2} \mathrm{O}\right), 4.07$ (1H, q, $\left.J=6.7, \mathrm{C} \underline{\mathrm{HOBn}}\right), 3.47$ $(1 \mathrm{H}, \mathrm{dd}, J=8.3, J=2.9, \mathrm{C} \underline{\mathrm{HOH}}), 3.25\left(1 \mathrm{H}, \mathrm{qd}, J=7.0, J=2.9, \mathrm{COC}_{\mathbf{H C H}}\right), 1.72-1.62(1 \mathrm{H}, \mathrm{m}$, $\left.\mathrm{CH}_{3} \mathrm{CHCH}_{3}\right), 1.38\left(3 \mathrm{H}, \mathrm{d}, J=6.7, \mathrm{CH}_{3} \mathrm{CHOBn}\right), 1.08\left(3 \mathrm{H}, \mathrm{d}, J=7.0, \mathrm{COCHCH}_{3}\right), 1.01(3 \mathrm{H}, \mathrm{d}, J$ $\left.=6.5, \mathrm{CH}_{3} \mathrm{CHCH}_{3}\right), 0.86\left(3 \mathrm{H}, \mathrm{d}, J=6.7, \mathrm{CH}_{3} \mathrm{CHCH}_{3}\right) .{ }^{13} \mathbf{C} \mathbf{N M R}\left(75.4 \mathrm{MHz}, \mathrm{CDCl}_{3}\right) \delta 216.9,137.5$, 128.5, 127.9, 127.8, 79.4, 76.4, 71.8, 42.6, 30.8, 19.0, 19.0, 16.8. 9.3. HRMS (+FAB): $\mathrm{m} / z$ calcd. for $\mathrm{C}_{16} \mathrm{H}_{25} \mathrm{O}_{3}[\mathrm{M}+\mathrm{H}]^{+}:$263.1804. Found: 263.1817.

\section{8. (2S,4S,5R)-2-Benzyloxy-5-hydroxy-4,7-dimethyl-3-octanone (5b).}

Colourless oil. $\boldsymbol{R}_{f}$ (hexanes/EtOAc 85:15) $=0.2$. HPLC (hexanes $\left./ i-\mathrm{PrOH} 99: 1\right) \boldsymbol{t}_{\mathbf{R}}=9.5 \mathrm{~min} .[\alpha]_{\mathrm{D}}=$ $+0.9\left(c=1.46, \mathrm{CHCl}_{3}\right)$. IR (film): v 3490 (br), 2958, 2871, 1713, 1455, $1113 \mathrm{~cm}^{-1}$. ${ }^{1} \mathbf{H}$ NMR $(500$

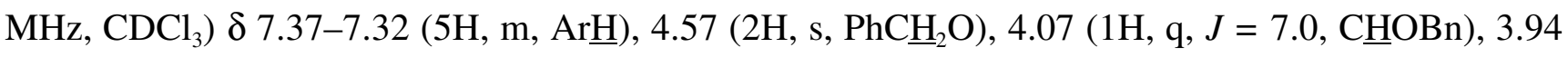
$(1 \mathrm{H}, \mathrm{ddd}, J=9.4, J=4.0, J=3.0, \mathrm{CHOH}), 3.03\left(1 \mathrm{H}, \mathrm{qd}, J=7.2, J=3.0, \mathrm{COC}^{\mathrm{HCH}}{ }_{3}\right), 1.80-1.72$ $\left(1 \mathrm{H}, \mathrm{m}, \mathrm{CH}_{3} \mathrm{CHCH}_{3}\right), 1.45\left(1 \mathrm{H}, \mathrm{ddd}, J=13.7, J=9.4, J=5.3, \mathrm{CH}(\mathrm{OH}) \underline{\mathrm{H}}_{x} \mathrm{H}_{\mathrm{y}}\right), 1.38(3 \mathrm{H}, \mathrm{d}, J=7.0$, $\left.\mathrm{C}_{3} \mathrm{CHOBn}\right), 1.10\left(3 \mathrm{H}, \mathrm{d}, J=7.2, \mathrm{COCHC}_{3}\right), 1.10(1 \mathrm{H}, \mathrm{ddd}, J=13.7, J=8.7, J=4.0$, $\left.\mathrm{CH}(\mathrm{OH}) \mathrm{CH}_{\mathrm{x}} \underline{\mathrm{H}}_{\mathrm{y}}\right), 0.92\left(3 \mathrm{H}, \mathrm{d}, J=6.7, \mathrm{CH}_{3} \mathrm{CHCH}_{3}\right), 0.90\left(3 \mathrm{H}, \mathrm{d}, J=6.7, \mathrm{CH}_{3} \mathrm{CHCH}_{3}\right) .{ }^{13} \mathbf{C} \mathbf{N M R}$ $\left(75.4 \mathrm{MHz}, \mathrm{CDCl}_{3}\right) \delta 216.5,137.4,128.5,127.9,127.8,79.5,71.7,69.3,45.7,43.1,24.6,23.4,21.9$, 16.5, 9.9. HRMS (+FAB): $m / z$ calcd. for $\mathrm{C}_{17} \mathrm{H}_{27} \mathrm{O}_{3}[\mathrm{M}+\mathrm{H}]^{+}: 279.1960$. Found: 279.1969 . 


\section{9. (2S,4S,5R)-2-Benzyloxy-5-hydroxy-4-methyl-3-heptanone (5c).}

Colourless oil. $\boldsymbol{R}_{f}\left(\right.$ hexanes/EtOAc 85:15) $=0.2$. HPLC $\left(\right.$ hexanes $/ i-\operatorname{PrOH~99:1)~} \boldsymbol{t}_{\mathbf{R}}=15.4 \mathrm{~min} .[\boldsymbol{\alpha}]_{\mathrm{D}}=$ -12.0 ( $c=1.25, \mathrm{CHCl}_{3}$ ). IR (film): v 3490 (br), 2975, 2937, 1715, 1457, $1104 \mathrm{~cm}^{-1}$. ${ }^{1} \mathbf{H}$ NMR (400 $\left.\mathrm{MHz}, \mathrm{CDCl}_{3}\right) \delta 7.40-7.30(5 \mathrm{H}, \mathrm{m}, \mathrm{Ar} \underline{\mathrm{H}}), 4.57\left(2 \mathrm{H}, \mathrm{s}, \mathrm{PhC}_{2} \mathrm{O}\right), 4.07(1 \mathrm{H}, \mathrm{q}, J=6.8, \mathrm{C} \underline{\mathrm{HOBn}})$, 3.80-3.70 (1H, m, CㅍH), $3.09\left(1 \mathrm{H}, \mathrm{qd}, J=7.1, J=3.1, \mathrm{COC}^{\mathrm{HCH}} \mathrm{H}_{3}\right), 1.60-1.35\left(2 \mathrm{H}, \mathrm{CH}_{2} \mathrm{CH}_{3}\right), 1.37$ $\left(3 \mathrm{H}, \mathrm{d}, J=6.8, \underline{\mathrm{C}}_{3} \mathrm{CHOBn}\right), 1.09\left(3 \mathrm{H}, \mathrm{d}, J=7.1, \mathrm{COCHC}_{3}\right), 0.95\left(3 \mathrm{H}, \mathrm{t}, J=7.4, \mathrm{CH}_{2} \underline{\mathrm{C}}_{3}\right) .{ }^{13} \mathrm{C}$ NMR $\left(100.6 \mathrm{MHz}, \mathrm{CDCl}_{3}\right) \delta 216.6,137.5,128.5,128.0,127.8,79.6,72.9,71.8,44.9,27.0,16.6$, $10.4,9.9$.

10. (2S,4S,5S)-2-Benzyloxy-5-hydroxy-4,6-dimethyl-6-hepten-3-one (5d).

Colourless oil. $\boldsymbol{R}_{f}$ (hexanes/EtOAc 85:15) $=0.3$. HPLC $\left(\right.$ hexanes $/ i-\operatorname{PrOH~99:1)~} \boldsymbol{t}_{\mathbf{R}}=10.8 \mathrm{~min} .[\alpha]_{\mathbf{D}}=$ -12.7 ( $c=1.07, \mathrm{CHCl}_{3}$ ). IR (film): v 3483 (br), 3033, 2983, 2937, 1715, 1654, 1455, $1119 \mathrm{~cm}^{-1} .{ }^{1} \mathbf{H}$ NMR $\left(500 \mathrm{MHz}, \mathrm{CDCl}_{3}\right) \delta$ 7.40-7.30 (5H, m, Arth), $5.07\left(1 \mathrm{H}\right.$, br s, $\left.\mathrm{C}\left(\mathrm{CH}_{3}\right)=\mathrm{C}_{\mathrm{x}} \mathrm{H}_{\mathrm{y}}\right), 4.94(1 \mathrm{H}$, br s, $\left.\mathrm{C}\left(\mathrm{CH}_{3}\right)=\mathrm{CH}_{x} \underline{\mathrm{H}}_{\mathrm{y}}\right), 4.57\left(2 \mathrm{H}, \mathrm{s}, \mathrm{PhC}_{2} \mathrm{O}\right), 4.38-4.37(1 \mathrm{H}, \mathrm{m}, \mathrm{C} \underline{\mathrm{HOH}}), 4.08(1 \mathrm{H}, \mathrm{q}, J=7.0, \mathrm{C} \underline{\mathrm{HOBn}})$, $3.24\left(1 \mathrm{H}, \mathrm{qd}, J=7.0, J=3.7, \mathrm{COC}^{\mathrm{HCH}}{ }_{3}\right), 1.69\left(3 \mathrm{H}\right.$, br s, $\left.\mathrm{C}\left(\mathrm{C}_{3}\right)=\mathrm{CH}_{2}\right), 1.40(3 \mathrm{H}, \mathrm{d}, J=7.0$, $\left.\mathrm{C}_{3} \mathrm{CHOBn}\right), 1.05\left(3 \mathrm{H}, \mathrm{d}, J=7.0, \mathrm{COCHC}_{3}\right) .{ }^{13} \mathbf{C ~ N M R}\left(75.4 \mathrm{MHz}, \mathrm{CDCl}_{3}\right) \delta 215.9,143.8,137.5$, 128.5, 127.9, 127.8, 112.0, 79.6, 74.0, 71.8, 43.2, 19.2, 16.7, 9.7. HRMS (+FAB): $\mathrm{m} / z$ calcd. for $\mathrm{C}_{16} \mathrm{H}_{23} \mathrm{O}_{3}[\mathrm{M}+\mathrm{H}]^{+}:$263.1647. Found: 263.1657.

\section{1. (1S,2S,4S)-4-Benzyloxy-1-hydroxy-2-methyl-1-phenyl-3-pentanone (5e).}

White solid. $\mathbf{m p}=56-57^{\circ} \mathrm{C} . \boldsymbol{R}_{f}$ (hexanes/EtOAc 85:15) = 0.2. HPLC (hexanes/i-PrOH 99:1) $\boldsymbol{t}_{\mathbf{R}}=$ $11.9 \min .[\alpha]_{\mathbf{D}}=+6.9\left(c=1.37, \mathrm{CHCl}_{3}\right)$. IR (KBr): $): v 3467$ (br), 3033, 2983, 2936, 1717, 1455, $1121 \mathrm{~cm}^{-1} .{ }^{1} \mathbf{H}$ NMR $\left(500 \mathrm{MHz}, \mathrm{CDCl}_{3}\right) \delta 7.40-7.20(10 \mathrm{H}, \mathrm{m}, \mathrm{Ar} \underline{\mathrm{H}}), 4.97(1 \mathrm{H}, \mathrm{d}, J=5.0, \mathrm{CHOH})$, $4.43\left(1 \mathrm{H}, \mathrm{d}, J=11.7, \mathrm{PhC}_{\mathrm{H}} \mathrm{H}_{\mathrm{y}} \mathrm{O}\right), 4.29\left(1 \mathrm{H}, \mathrm{d}, J=11.7, \mathrm{PhCH}_{x} \underline{H}_{\mathrm{y}} \mathrm{O}\right), 3.88(1 \mathrm{H}, \mathrm{q}, J=7.0, \mathrm{C} \underline{\mathrm{HOBn}})$, $3.30\left(1 \mathrm{H}, \mathrm{qd}, J=7.0, J=5.0, \mathrm{COCHCH}_{3}\right), 1.26\left(3 \mathrm{H}, \mathrm{d}, J=7.0, \mathrm{CH}_{3} \mathrm{CHOBn}\right), 1.11$ (3H, d, $J=7.0$, $\left.\mathrm{COCHCH}_{3}\right) .{ }^{13} \mathbf{C ~ N M R}\left(75.4 \mathrm{MHz}, \mathrm{CDCl}_{3}\right) \delta 215.7,141.8,137.5,128.4,128.3,127.8,127.7,127.6$, 126.3, 80.1, 73.9, 71.7, 48.1, 16.4, 11.2. HRMS (+FAB): $m / z$ calcd. for $\mathrm{C}_{19} \mathrm{H}_{23} \mathrm{O}_{3}[\mathrm{M}+\mathrm{H}]^{+}: 299.1647$. Found: 299.1636. 
12. (1S,2S,4S)-4-Benzyloxy-1-(p-chlorophenyl)-1-hydroxy-2-methyl-3-pentanone (5f).

White solid. $\mathbf{m p}=66-68{ }^{\circ} \mathrm{C} . \boldsymbol{R}_{f}\left(\right.$ hexanes/EtOAc 85:15) $=0.2$. HPLC $\left(\right.$ hexanes $/ i-\operatorname{PrOH}$ 99.5:0.5) $\boldsymbol{t}_{\mathbf{R}}=$ $24.5 \min .[\alpha]_{\mathbf{D}}=-2.0\left(c=1.07, \mathrm{CHCl}_{3}\right)$. IR (KBr): v 3467 (br), 3033, 2983, 2937, 1713, 1493, 1455, $1092 \mathrm{~cm}^{-1} .{ }^{1} \mathbf{H}$ NMR $\left(300 \mathrm{MHz}, \mathrm{CDCl}_{3}\right) \delta 7.40-7.20(9 \mathrm{H}, \mathrm{m}, \mathrm{Ar} \underline{\mathrm{H}}), 4.98(1 \mathrm{H}, \mathrm{dd}, J=4.2, J=1.8$, $\mathrm{C} \underline{\mathrm{HOH}}), 4.49\left(1 \mathrm{H}, \mathrm{AB}\right.$ system, $\left.J=11.6, \mathrm{PhC}_{\mathrm{H}} \mathrm{H}_{\mathrm{y}} \mathrm{O}\right), 4.42\left(1 \mathrm{H}, \mathrm{AB}\right.$ system, $\left.J=11.6, \mathrm{PhCH}_{x} \underline{H}_{y} \mathrm{O}\right)$, $3.93(1 \mathrm{H}, \mathrm{q}, J=6.9, \mathrm{CHOBn}), 3.29\left(1 \mathrm{H}, \mathrm{qd}, J=7.1, J=4.2, \mathrm{COCHCH}_{3}\right), 1.29$ (3H, d, $J=6.9$, $\left.\mathrm{C}_{3} \mathrm{CHOBn}\right), 1.05\left(3 \mathrm{H}, \mathrm{d}, J=7.1, \mathrm{COCHCH}_{3}\right) .{ }^{13} \mathbf{C ~ N M R}\left(75.4 \mathrm{MHz}, \mathrm{CDCl}_{3}\right) \delta 215.9,140.2,137.3$, 133.2, 128.5, 128.4, 128.0, 127.8, 127.6, 79.8, 72.9, 71.8, 47.7, 16.3, 10.7. HRMS (+FAB): $\mathrm{m} / \mathrm{z}$ calcd. for $\mathrm{C}_{19} \mathrm{H}_{22} \mathrm{ClO}_{3}[\mathrm{M}+\mathrm{H}]^{+}:$333.1257. Found: 333.1262 .

13. (2S,4R,5R)-2-Benzyloxy-5-hydroxy-4,6-dimethyl-6-hepten-3-one (6d).

Colourless oil. $\boldsymbol{R}_{f}$ (hexanes/EtOAc 85:15) $=0.3$. HPLC (hexanes/i-PrOH 99:1) $\boldsymbol{t}_{\mathbf{R}}=9.4 \mathrm{~min} .[\boldsymbol{\alpha}]_{\mathrm{D}}=$ -27.1 (c = 1.37, $\mathrm{CHCl}_{3}$ ). IR (film): v 3490 (br), 3033, 2983, 2939, 1717, 1654, 1457, $1121 \mathrm{~cm}^{-1} .{ }^{1} \mathbf{H}$ NMR $\left(500 \mathrm{MHz}, \mathrm{CDCl}_{3}\right) \delta 7.40-7.30(5 \mathrm{H}, \mathrm{m}, \mathrm{Ar} \underline{\mathrm{H}}), 5.09-5.08\left(1 \mathrm{H}, \mathrm{m}, \mathrm{C}\left(\mathrm{CH}_{3}\right)=\mathrm{C}_{\mathrm{x}} \mathrm{H}_{\mathrm{y}}\right), 4.95-4.94$ $\left(1 \mathrm{H}, \mathrm{m}, \mathrm{C}\left(\mathrm{CH}_{3}\right)=\mathrm{CH}_{\mathrm{x}} \underline{\mathrm{H}}_{\mathrm{y}}\right), 4.57\left(2 \mathrm{H}, \mathrm{s}, \mathrm{PhCH}_{2} \mathrm{O}\right), 4.37(1 \mathrm{H}$, br s, $\mathrm{CHOH}), 4.09(1 \mathrm{H}, \mathrm{q}, J=7.0$,

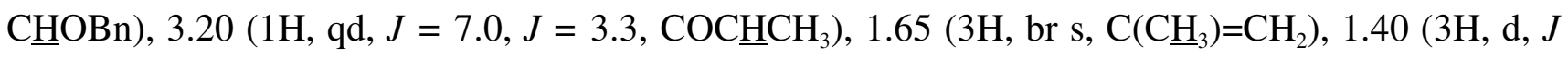
=7.0, $\left.\mathrm{C}_{3} \mathrm{CHOBn}\right), 1.06$ (3H, d, $\left.J=7.0, \mathrm{COCHC}_{3}\right) .{ }^{13} \mathbf{C ~ N M R}\left(75.4 \mathrm{MHz}, \mathrm{CDCl}_{3}\right) \delta$ 216.4, 143.6, 137.4, 128.5, 127.9, 127.7, 111.8, 79.5, 73.6, 71.7, 43.1, 19.3, 17.1, 9.8. HRMS (+FAB): $\mathrm{m} / z$ calcd. for $\mathrm{C}_{16} \mathrm{H}_{23} \mathrm{O}_{3}[\mathrm{M}+\mathrm{H}]^{+}:$263.1647. Found: 263.1644.

\section{4. (1R,2R,4S)-4-Benzyloxy-1-hydroxy-2-methyl-1-phenyl-3-pentanone (6e).}

White oil. $\boldsymbol{R}_{f}$ (hexanes/EtOAc 75:25) = 0.2. HPLC (hexanes/i-PrOH 99:1) $\boldsymbol{t}_{\mathbf{R}}=8.7 \mathrm{~min} .[\boldsymbol{\alpha}]_{\mathrm{D}}=-33.2$ $\left(c=1.93, \mathrm{CHCl}_{3}\right)$. IR (film): v 3475 (br), 3033, 2988, 2937, 1717, 1457, $1109 \mathrm{~cm}^{-1}$. ${ }^{1} \mathbf{H}$ NMR $(250$ $\left.\mathrm{MHz} \mathrm{CDCl}_{3}\right) \delta$ 7.36-7.25 (10H, m, ArH $), 5.01(1 \mathrm{H}, \mathrm{d}, J=4.5, \mathrm{C} \underline{\mathrm{HOH}}), 4.49(1 \mathrm{H}, \mathrm{AB}$ system, $J=$ 11.7, $\left.\mathrm{PhC} \underline{H}_{x} \mathrm{H}_{\mathrm{y}} \mathrm{O}\right), 4.43\left(1 \mathrm{H}, \mathrm{AB}\right.$ system, $\left.J=11.7, \mathrm{PhCH}_{\mathrm{x}} \underline{\mathrm{H}}_{\mathrm{y}} \mathrm{O}\right), 3.93(1 \mathrm{H}, \mathrm{q}, J=6.9, \mathrm{C} \underline{\mathrm{HOBn}}), 3.24$ $\left(1 \mathrm{H}, \mathrm{qd}, J=7.2, J=4.5, \mathrm{COC}^{\mathrm{HCH}}{ }_{3}\right), 1.24\left(3 \mathrm{H}, \mathrm{d}, J=6.9, \mathrm{C}_{3} \mathrm{CHOBn}\right), 1.10$ (3H, d, $J=7.2$, COCHCH$\left.\underline{H}_{3}\right) .{ }^{13} \mathbf{C ~ N M R}\left(75.4 \mathrm{MHz}, \mathrm{CDCl}_{3}\right) \delta 216.3,141.8,137.4,128.4,128.2,127.9,127.7,127.3$, 126.0, 79.5, 73.2, 71.6, 48.2, 16.9, 11.1. HRMS (+FAB): $\mathrm{m} / z$ calcd. for $\mathrm{C}_{19} \mathrm{H}_{23} \mathrm{O}_{3}[\mathrm{M}+\mathrm{H}]^{+}: 299.1647$. Found: 299.1655. 


\section{5. (1R,2R,4S)-4-Benzyloxy-1-(p-chlorophenyl)-1-hydroxy-2-methyl-3-pentanone (6f)}

White oil. $\boldsymbol{R}_{f}$ (hexanes/EtOAc 85:15) $=0.2$. HPLC (hexanes $\left./ i-\mathrm{PrOH} 99: 1\right) \boldsymbol{t}_{\mathbf{R}}=10.9 \mathrm{~min} .[\boldsymbol{\alpha}]_{\mathrm{D}}=$ -34.0 ( $c=1.29, \mathrm{CHCl}_{3}$ ). IR (film): v 3473 (br), 3033, 2983, 2935, 1713, 1493, 1455, $1090 \mathrm{~cm}^{-1} .{ }^{1} \mathbf{H}$ NMR (400 MHz, $\left.\mathrm{CDCl}_{3}\right) \delta$ 7.38-7.19 (9H, m, Ar $\left.\underline{\mathrm{H}}\right), 4.99(1 \mathrm{H}$, br s, $\underline{\mathrm{CHOH}}), 4.50\left(2 \mathrm{H}, \mathrm{s}, \mathrm{PhC}_{2} \mathrm{O}\right)$, $3.97(1 \mathrm{H}, \mathrm{q}, J=6.8, \mathrm{C} \underline{\mathrm{HOBn}}), 3.21\left(1 \mathrm{H}, \mathrm{qd}, J=7.0, J=4.1, \mathrm{COC}^{\mathrm{HCH}}{ }_{3}\right), 1.30(3 \mathrm{H}, \mathrm{d}, J=6.8$, $\left.\mathrm{C}_{3} \mathrm{CHOBn}\right), 1.06\left(3 \mathrm{H}, \mathrm{d}, J=7.0, \mathrm{COCHCH}_{3}\right) .{ }^{13} \mathrm{C} \mathrm{NMR}\left(100.6 \mathrm{MHz}, \mathrm{CDCl}_{3}\right) \delta 216.5,140.2$, $137.3,133.1,128.6,128.4,128.0,127.8,127.4,79.6,72.5,71.7,47.8,17.1,10.7$.

\section{2. ${ }^{1} H$ NMR copies.}




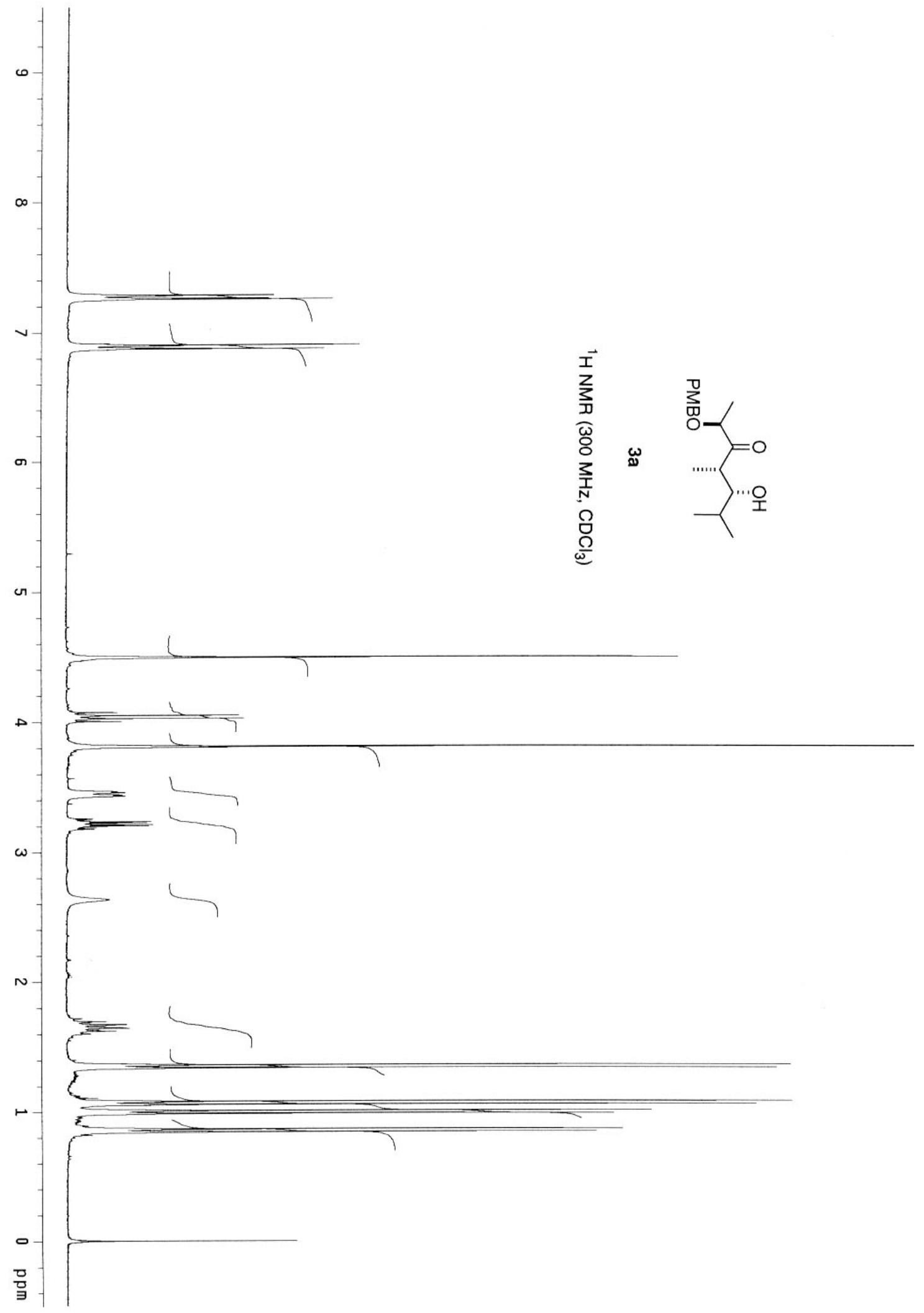




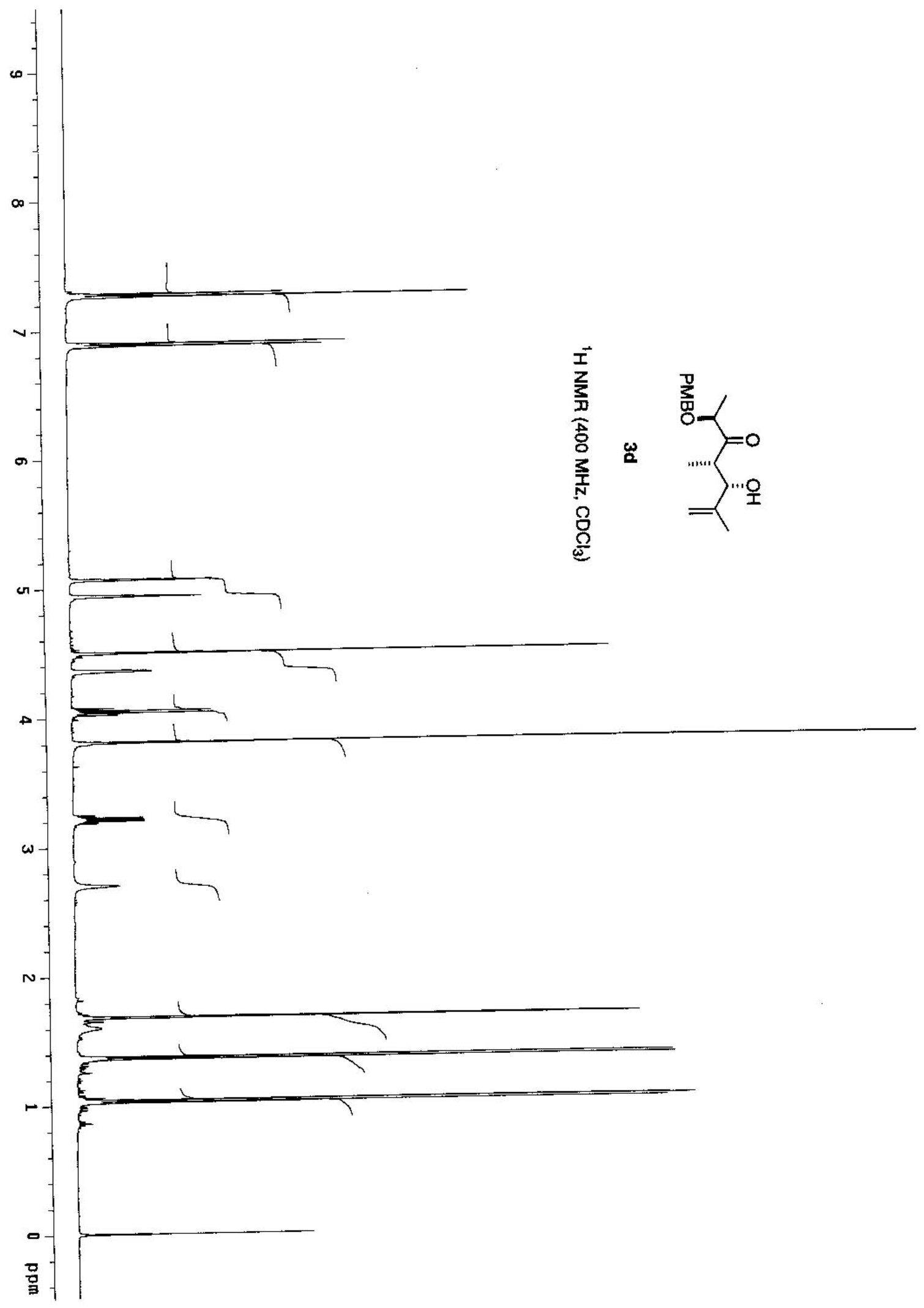

8 


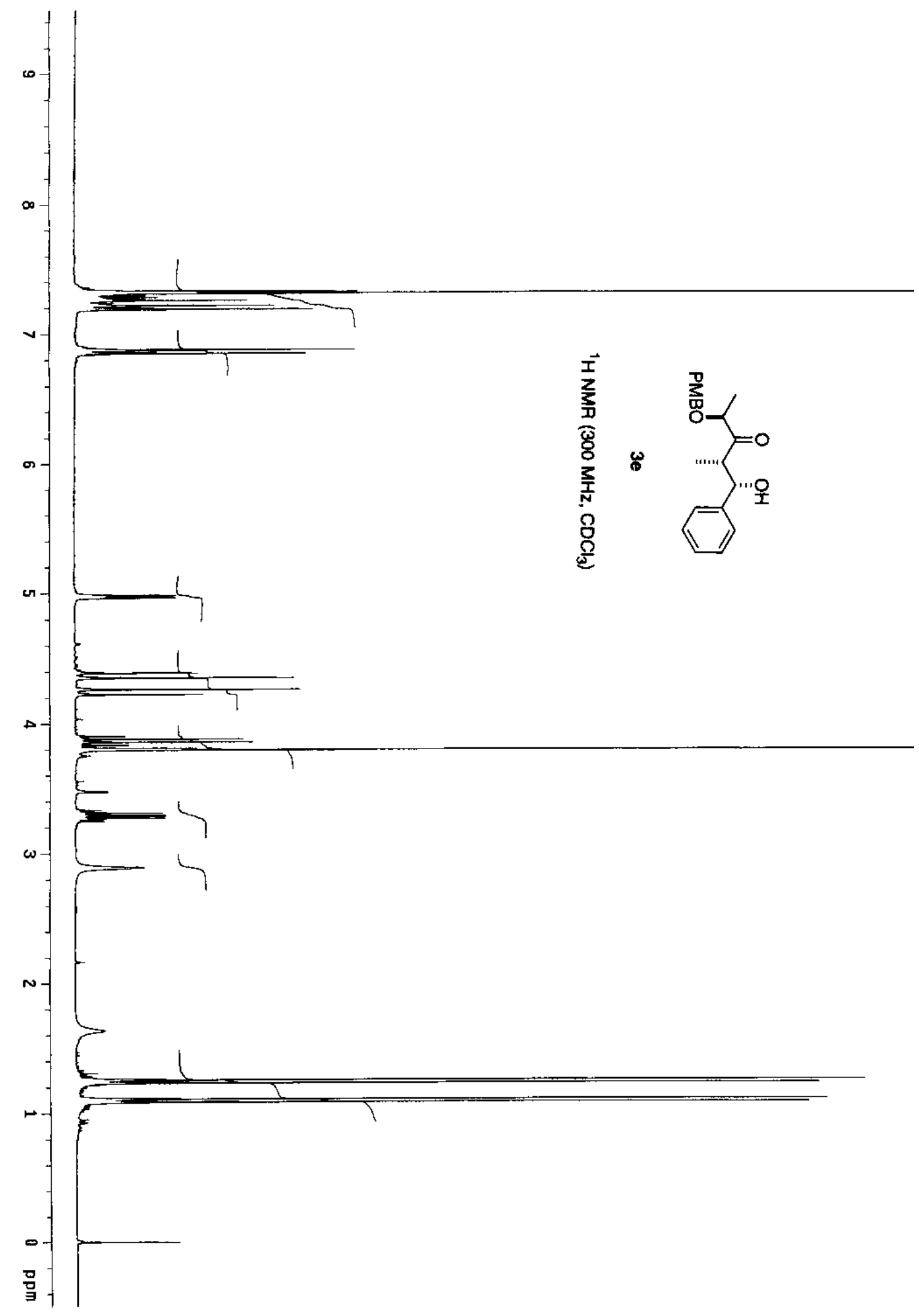




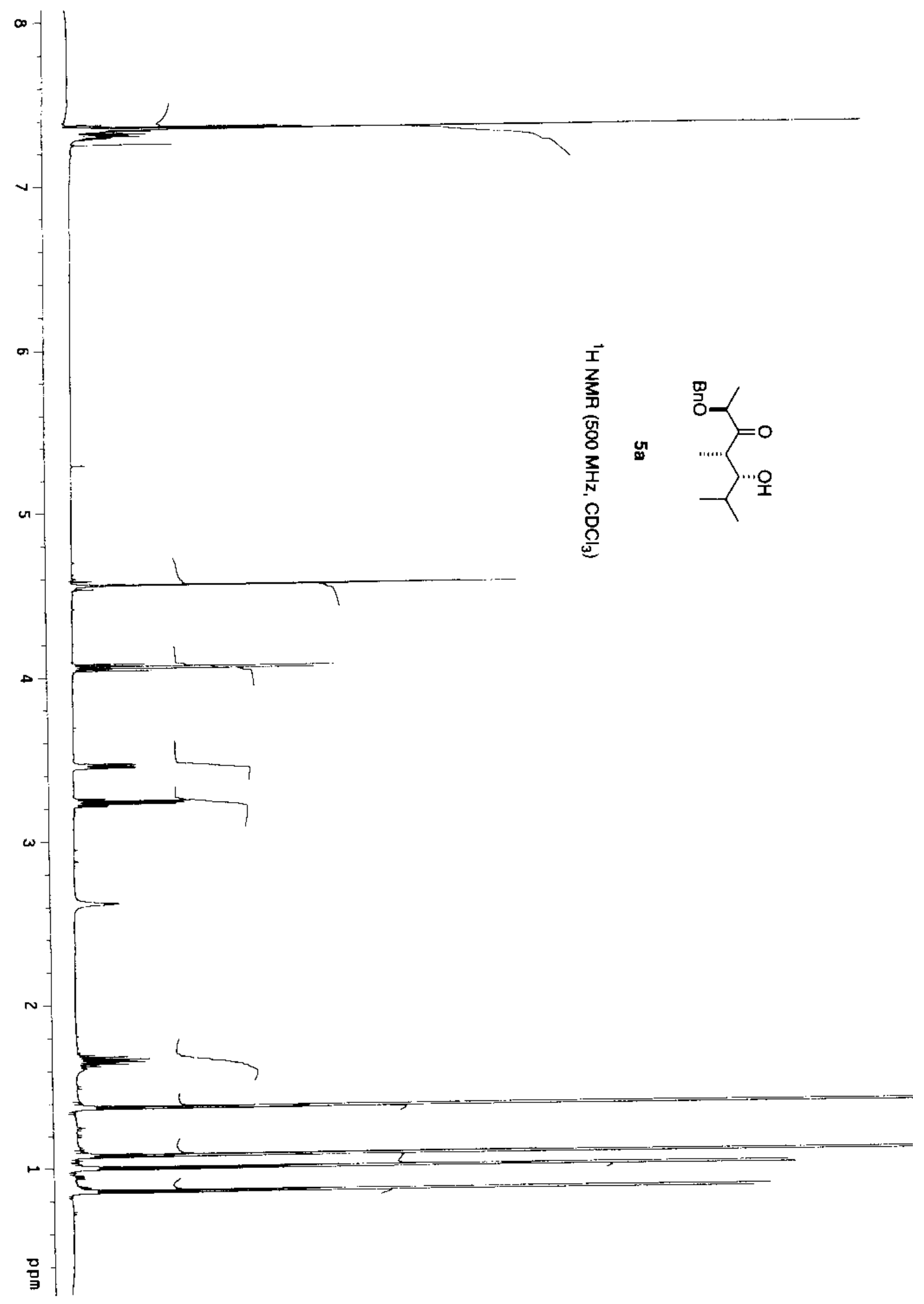




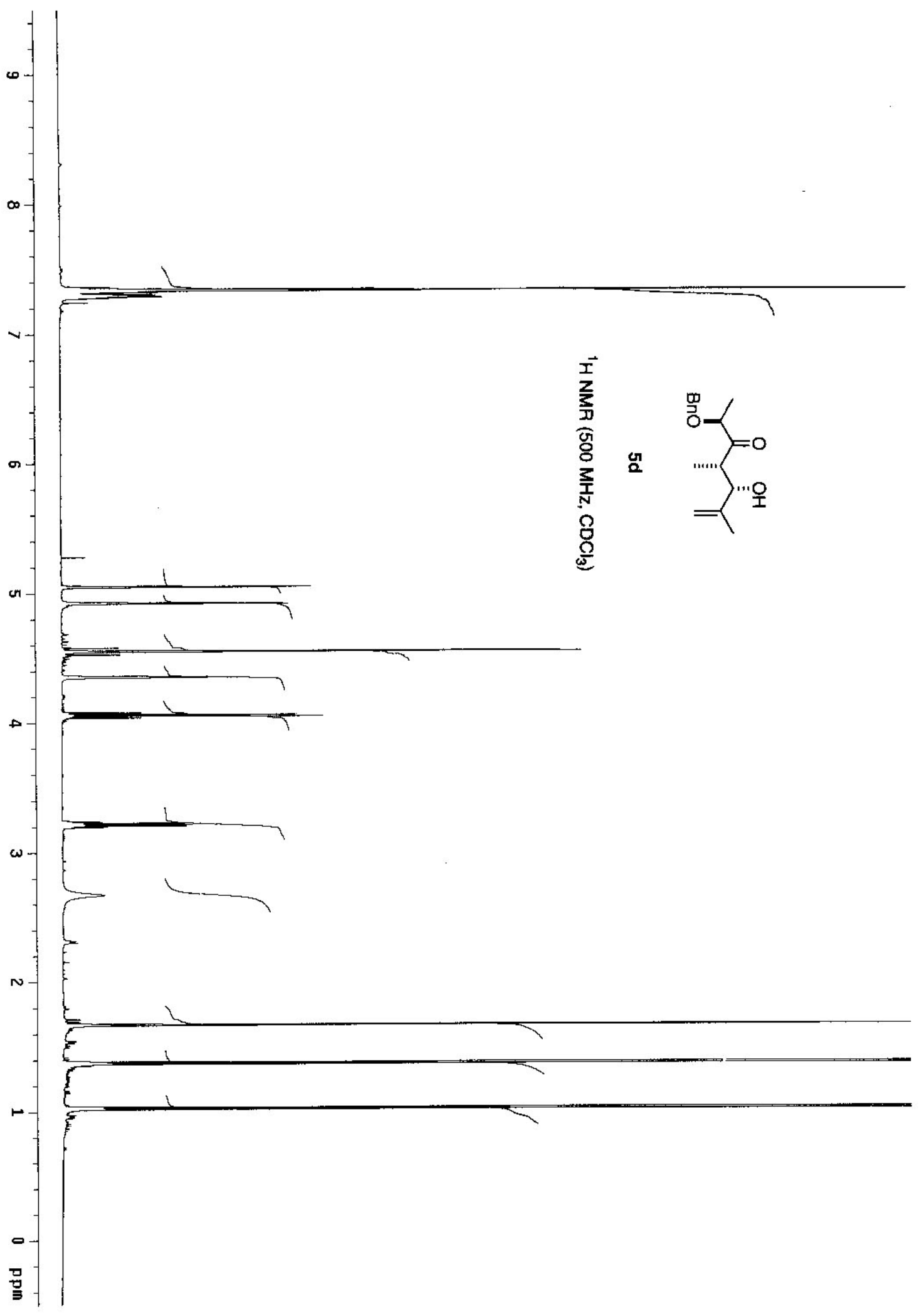




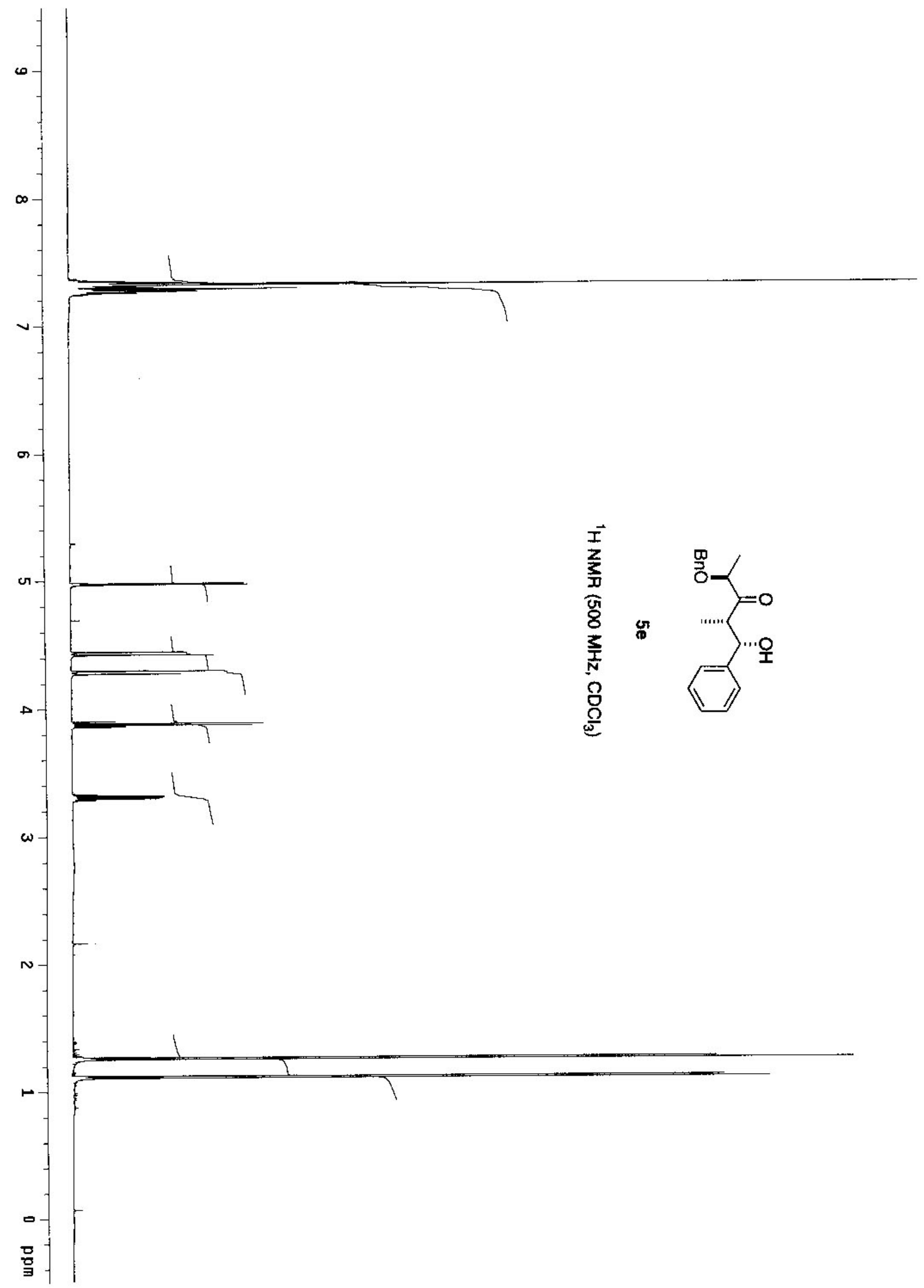




\section{Crystal data for 3 f and $5 f$.}

1. (1S,2S,4S)-1-(p-Chlorophenyl)-1-hydroxy-4-(p-methoxybenzyloxy)-2-methyl-3-pentanone (3f)

Empirical formula

Formula weight

Temperature

Wavelength

Crystal system, space group

Unit cell dimensions

Volume

Z, Calculated density

Absorption coefficient

$\mathrm{F}(000)$

Crystal size

Theta range for data collection

Index ranges

Reflections collected / unique

Refinement method

Data / restraints / parameters

Goodness-of-fit on $\mathrm{F}^{2}$

Final $R$ indices $[\mathrm{I}>2 \sigma(\mathrm{I})]$

$\mathrm{R}$ indices (all data)

Absolute structure parameter

Largest diff. peak and hole
$\mathrm{C}_{20} \mathrm{H}_{23} \mathrm{ClO}_{4}$

362.83

$571(2) \mathrm{K}$

$0.71069 \AA$

Monoclinic, $\mathrm{P} 2_{1}$

$\mathrm{a}=11.069$ (4) $\AA \quad \alpha=90^{\circ}$

$\mathrm{b}=8.021(8) \AA \quad \beta=116.87(2)^{\circ}$

$\mathrm{c}=12.096(2) \AA \quad \gamma=90^{\circ}$

$958.0(10) \AA^{3}$

2, $1.258 \mathrm{Mg} / \mathrm{m}^{3}$

$0.220 \mathrm{~mm}^{-1}$

384

$0.1 \times 0.1 \times 0.2 \mathrm{~mm}$

2.06 to $29.96^{\circ}$

$-15<=\mathrm{h}<=13,-3<=\mathrm{k}<=11,0<=\mathrm{l}<=17$

$3087 / 2956[\mathrm{R}(\mathrm{int})=0.0253]$

Full-matrix least-squares on $\mathrm{F}^{2}$

2956 / 1 / 268

1.041

$\mathrm{R} 1=0.0580, \mathrm{wR} 2=0.1398$

$\mathrm{R} 1=0.1006, \mathrm{wR} 2=0.1596$

0.08 (14)

0.348 and -0.282 e. $\AA^{-3}$ 


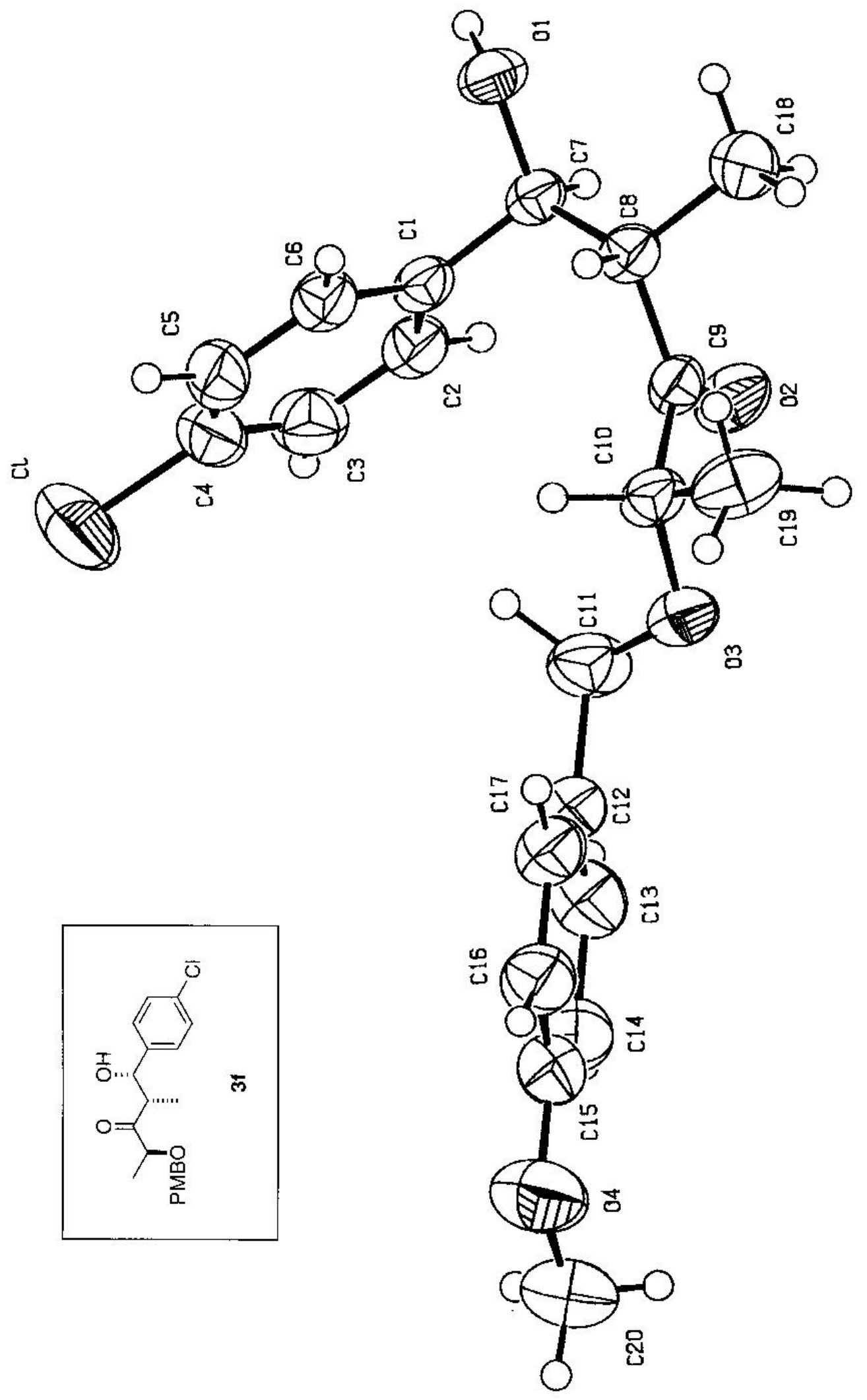




\section{2. (1S,2S,4S)-4-Benzyloxy-1-(p-chlorophenyl)-1-hydroxy-2-methyl-3-pentanone (5f)}

Empirical formula

Formula weight

Temperature

Wavelength

Crystal system, space group

Unit cell dimensions

Volume

Z, Calculated density

Absorption coefficient

$\mathrm{F}(000)$

Crystal size

Theta range for data collection

Index ranges

Reflections collected / unique

Refinement method

Data / restraints / parameters

Goodness-of-fit on $\mathrm{F}^{2}$

Final R indices [I $>2 \sigma(\mathrm{I})]$

$\mathrm{R}$ indices (all data)

Absolute structure parameter

Extiction coefficient

Largest diff. peak and hole
$\mathrm{C}_{19} \mathrm{H}_{21} \mathrm{ClO}_{3}$

332.81

293 (2) K

$0.71069 \AA$

Orthorhombic, P2 $2_{1} 2_{1}$

$\mathrm{a}=8.3420(10) \AA \quad \alpha=90^{\circ}$

$\mathrm{b}=10.8600$ (10) $\AA \quad \beta=90^{\circ}$

$\mathrm{c}=19.3020$ (10) $\AA \quad \gamma=90^{\circ}$

$1748.6(3) \AA^{3}$

$4,1.264 \mathrm{Mg} / \mathrm{m}^{3}$

$0.230 \mathrm{~mm}^{-1}$

704

$0.1 \times 0.1 \times 0.2 \mathrm{~mm}$

2.66 to $24.88^{\circ}$

$0<=\mathrm{h}<=9,0<=\mathrm{k}<=10,0<=\mathrm{l}<=22$

$7573 / 1490$ [R(int) $=0.0437]$

Full-matrix least-squares on $\mathrm{F}^{2}$

1490 / 0 / 293

1.077

$\mathrm{R} 1=0.0417, \mathrm{wR} 2=0.1043$

$\mathrm{R} 1=0.0666, \mathrm{wR} 2=0.1147$

0.34 (18)

$0.011(3)$

0.155 and -0.171 e. $\AA^{-3}$ 

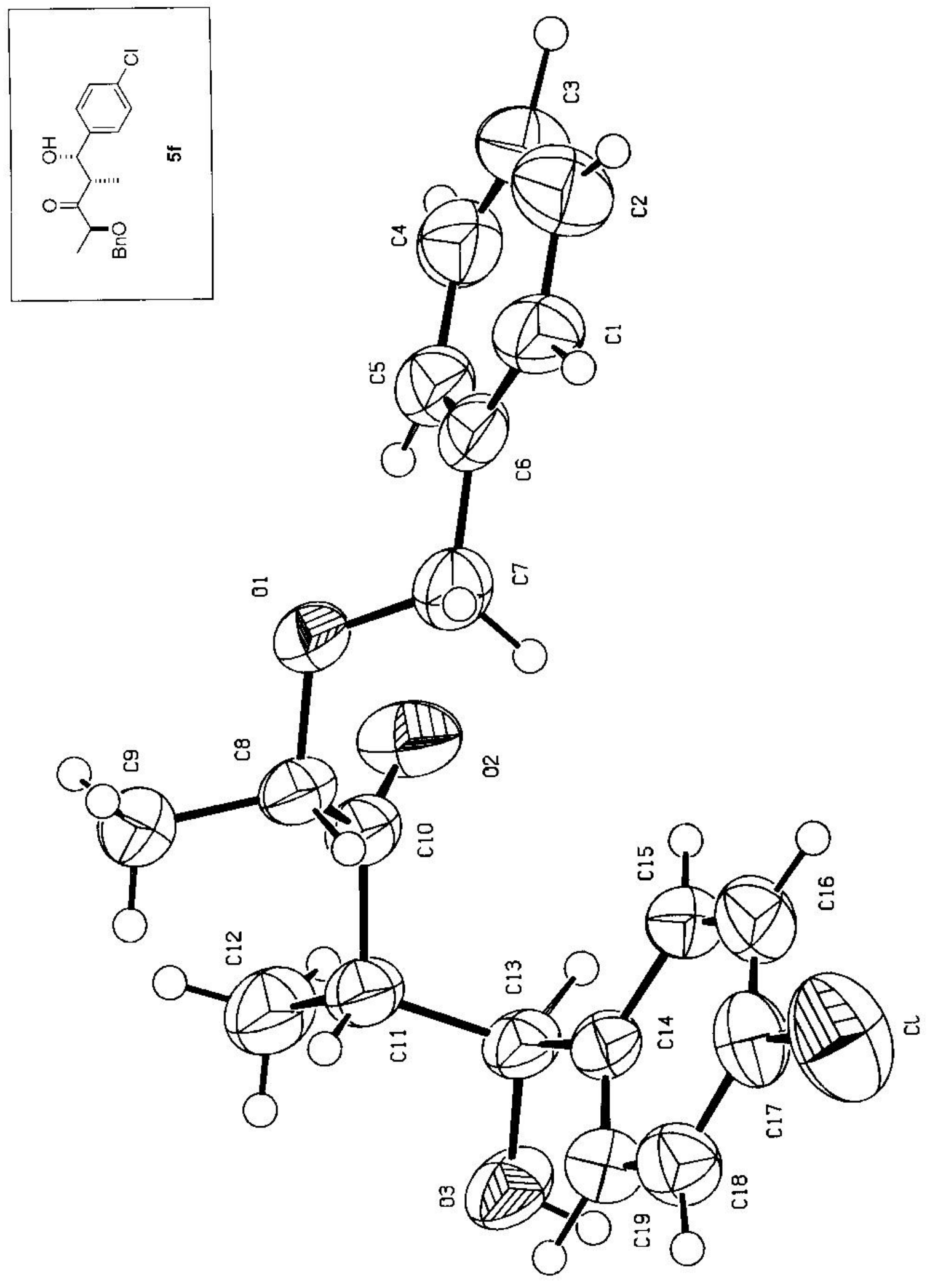